\title{
Age-dependent susceptibility to reovirus encephalitis in mice is influenced by maturation of the type-I interferon response
}

\author{
Allen G Wu ${ }^{1,2,6,7}$, Andrea J Pruijssers ${ }^{1,2,6}$, Judy J Brown ${ }^{2,3}$, Jennifer E Stencel-Baerenwald ${ }^{2,3,8}$, Danica M Sutherland ${ }^{2,3}$, \\ Jason A Iskarpatyoti ${ }^{1,2,9}$ and Terence $S$ Dermody ${ }^{4,5}$
}

BACKGROUND: Infants and young children are particularly susceptible to viral encephalitis; however, the mechanisms are unknown. We determined the age-dependent contribution of innate and adaptive immune functions to reovirus-induced encephalitis in mice.

METHODS: Newborn wild-type mice, 2-20 days of age, were inoculated with reovirus or diluent and monitored for mortality, weight gain, and viral load. Four- and fifteen-dayold $\mathrm{FNAR}^{-1-}$ and $\mathrm{RAG}^{-/-}$mice were inoculated with reovirus and similarly monitored.

RESULTS: Weight gain was impaired in mice inoculated with reovirus at 8 days of age or less. Clinical signs of encephalitis were detected in mice inoculated at 10 days of age or less. Mortality decreased when mice were inoculated after 6 days of age. Survival was $\leq 15 \%$ in wild type $(\mathrm{WT}), \mathrm{RAG}^{-1-}$, and IFNAR $^{-1-}$ mice inoculated at 4 days of age. All WT mice, $92 \%$ of $\mathrm{RAG}^{-1-}$ mice, and only $48 \%$ of IFNAR ${ }^{-1-}$ mice survived following inoculation at 15 days of age.

CONCLUSIONS: Susceptibility of mice to reovirus-induced disease decreases between 6 and 8 days of age. Enhanced reovirus virulence in $\mathrm{FNAR}^{-1-}$ mice relative to $W T$ and $\mathrm{RAG}^{-1-}$ mice inoculated at 15 days of age suggests that maturation of the type-l interferon response contributes to age-related mortality following reovirus infection.

\footnotetext{
V
} iral encephalitis is a major cause of morbidity and mortality worldwide. Neurotropic viruses continue to emerge and reemerge because of changes in viral virulence, expanded distribution of viral vectors, fluctuations in population immunity, and increased travel associated with globalization (1). The young are particularly susceptible to poor outcomes of viral encephalitis such as developmental delays, learning disabilities, epilepsy, and death. Neurotropic viruses replicate more efficiently and display enhanced apoptosis capacity in immature vs. mature neurons through mechanisms that are incompletely understood (2).

Reovirus serves as a highly tractable experimental system in which to study neurotropic virus-host interactions. Following peroral inoculation of newborn mice, reovirus infects the intestine and disseminates systemically to the mesenteric lymph nodes, liver, spleen, lungs, heart, and brain. Serotype 1 reovirus strains disseminate exclusively via hematogenous routes, whereas serotype 3 strains disseminate via hematogenous and neural routes $(3,4)$. Upon entry into the brain, serotype 3 strains infect neurons located in the frontal and parietal cortex, CA1 to CA4 regions of the hippocampus, the cingulate gyrus, the thalamus, and Purkinje neurons in the cerebellum (5,6). Reovirus infection of neurons causes apoptosis and triggers inflammation, resulting in a lethal meningoencephalitis (3,7-9).

The susceptibility of mice to reovirus infection and death is age-dependent. Infection of young mice with serotype 3 reovirus leads to a lethal encephalitis and death, whereas signs of clinical disease are absent following infection of older mice $(10,11)$. One study suggests that the age susceptibility of mice to reovirus neurovirulence is due to the inhibition of viral replication by neuronal cell intrinsic factors (12). However, the precise mechanism is unknown.

The type-I interferon (IFN) pathway is a major component of the innate immune system. Following production and secretion, IFNs act in an autocrine and paracrine manner to induce an antiviral state in infected and neighboring cells, promote a balanced natural killer cell response with controlled anti-inflammatory activities, and enhance antigen presentation to activate the adaptive immune system (13). Mice deficient in the signal transducer and activator of transcription-1, a key component of IFN signaling, display increased mortality and higher viral loads in the brain following intracranial inoculation with reovirus at 2 days of

\footnotetext{
${ }^{1}$ Department of Pediatrics, Vanderbilt University School of Medicine, Nashville, Tennessee; ${ }^{2}$ Department of Elizabeth B. Lamb Center for Pediatric Research, Vanderbilt University School of Medicine, Nashville, Tennessee; ${ }^{3}$ Department of Pathology, Microbiology, and Immunology, Vanderbilt University School of Medicine, Nashville, Tennessee; ${ }^{4}$ Department of Pediatrics, University of Pittsburgh School of Medicine, Pittsburgh, Pennsylvania; ${ }^{5}$ Department of Microbiology and Molecular Genetics, University of Pittsburgh School of Medicine, Pittsburgh, Pennsylvania. Correspondence: Terence S. Dermody (terence.dermody@chp.edu)

${ }^{6}$ These authors contributed equally to this work.

${ }^{7}$ Current address: Advocate Christ Medical Center Oak Lawn, Chicago, Illinois

${ }^{8}$ Current address: Biogen, Cambridge, Massachusetts

${ }^{9}$ Current address: Department of Molecular Genetics and Microbiology, Duke University School of Medicine, Durham, North Carolina

Received 5 September 2017; accepted 12 January 2018; advance online publication 21 February 2018. doi:10.1038/pr.2018.13
} 
age, suggesting that IFN signaling reduces reovirus replication and virulence (14).

Strategies to prevent and treat viral encephalitis are limited in part because of a lack of knowledge of the cellular factors and molecular mechanisms that contribute to viral virulence. We hypothesized that the susceptibility to reovirus disease is an effect of age-dependent control of the virus by maturing components of the immune system. To test this hypothesis, we defined the age at which mice lose susceptibility to reovirus-induced disease, determined the relationship between viral replication in vital organs and disease severity, and delineated age-dependent contributions from innate and adaptive immune functions. As age restriction is a shared determinant of disease severity in many neurotropic virus infections, it is possible that mechanisms underlying reovirus age restriction will be applicable to other viral infections.

\section{METHODS}

\section{Cell Lines and Viruses}

Murine L929 cells were maintained in Eagle's minimal essential medium (Lonza; Walkersville, MD) with 5\% fetal bovine serum (Gibco; Gaitersburg, MD), 2 mM L-glutamine (Invitrogen; Carlsbad, $\mathrm{CA}), 100 \mathrm{U} / \mathrm{ml}$ penicillin (Invitrogen), $100 \mu \mathrm{g} / \mathrm{ml}$ streptomycin (Invitrogen), and $25 \mathrm{ng} / \mathrm{ml}$ amphotericin B (Sigma-Aldrich; St. Louis, MO). Reovirus strain type 3 Dearing (T3D) is a laboratory stock recovered by plasmid-based reverse genetics (15). Reovirus was amplified in L929 cells and purified by Vertrel-XF (Dupont; Wilmington, DE) extraction, followed by CsCl-gradient centrifugation (16). Viral plaque-forming unit (PFU) titers were determined by plaque assay using L929 cells (17).

\section{Mouse Strains}

C57BL/6J (WT) mice were obtained from The Jackson Laboratory. C57BL/6 IFNAR ${ }^{-1-}$ mice were provided by John Williams (Vanderbilt University School of Medicine; Nashville, TN), and C57BL/6 RAG2 ${ }^{-/-}$ mice were provided by Danyvid Olivares-Villagomez (Vanderbilt University School of Medicine; Nashville, TN).

\section{Infection of Mice}

Mice were weighed and inoculated intracranially (18) with purified reovirus T3D in phosphate-buffered saline (PBS) at $100 \mathrm{PFU} / g$. For analysis of virulence, mice were monitored for weight gain and symptoms of disease for 20 days post inoculation (d p.i.) and killed when moribund. For analysis of viral replication, organs were collected into $1 \mathrm{ml}$ of PBS and homogenized using a TissueLyser (Qiagen; Hilden, Germany). Viral titers were determined by plaque assay. Animal husbandry and experimental procedures were performed in accordance with Public Health Service policy and approved by the Vanderbilt University School of Medicine Institutional Animal Care and Use Committee.

\section{Histology}

Mice were inoculated intracranially with reovirus T3D at $100 \mathrm{PFU} / \mathrm{g}$. Brains were resected and divided sagittally. Left-brain hemispheres were processed for plaque assay. Right-brain hemispheres were fixed in $10 \%$ formalin and embedded in paraffin. Consecutive $6-\mu \mathrm{m}$ sections were stained with hematoxylin and eosin or processed for immunohistochemical detection of reovirus antigen or the cleaved (active) form of caspase-3 (ref. 19).

\section{Immunoblotting}

Brain homogenates were diluted twofold in RIPA lysis buffer (SigmaAldrich) containing Complete Protease Inhibitor Cocktail (Roche; Basel, Switzerland). Protein extract $(50 \mu \mathrm{g})$ was resolved by electrophoresis in 10\% Tris-glycine gels (Bio-Rad; Hercules, CA) and transferred to Immun-Blot PVDF membranes (Bio-Rad). Membranes were blocked for at least $1 \mathrm{~h}$ in Odyssey blocking buffer (LI-COR; Lincoln, NE) and incubated with an anti-actin antibody (1:500; Santa Cruz Biotechnologies; Dallas, TX) and a cleaved (active) caspase-3 antibody (1:1,000; Cell Signaling Technologies; Danvers, MA) in Odyssey blocking buffer overnight. Membranes were washed and incubated with secondary antibodies IRDye $680 \mathrm{CW}$-conjugated donkey anti-goat $(1: 2,000)$ and IRDye $800 \mathrm{CW}$ conjugated goat anti-rabbit $(1: 5,000)$ in Odyssey blocking buffer for $2 \mathrm{~h}$. Membranes were washed three times and scanned using an Odyssey infrared imaging system (LI-COR). Signal intensities of specific bands were quantified using ImageStudio software (LI-COR).

\section{RESULTS}

\section{Survival of Mice from Reovirus Infection Is Age-Dependent}

Following infection with a neurotropic serotype 3 strain of reovirus, neonatal mice will contract encephalitis, which is often fatal, whereas adult mice do not develop overt signs of the disease $(10,11)$. To determine the age window during which WT mice become refractory to reovirus-induced mortality, mice were inoculated intracranially with $100 \mathrm{PFU} / \mathrm{g}$ T3D in PBS or PBS alone (mock) at 2, 4, 6, 8, 10, 15, and $20 \mathrm{~d}$ of age and monitored for survival for $20 \mathrm{~d}$. Only $37 \%$ of 2 -dayold $(n=16), 15 \%$ of 4 -day-old $(n=20)$, and $48 \%$ of 6 -day-old $(n=27)$ mice survived (Figure 1a). In contrast, $90 \%$ of mice inoculated with reovirus at $8 \mathrm{~d}$ of age $(n=18)$ and all mice inoculated at 10,15 , and $20 \mathrm{~d}$ of age $(n \geq 15)$ survived. Mice that became ill exhibited clinical signs of encephalitis including lethargy, seizures, ataxia, and paralysis. All PBS-inoculated mice survived. These data indicate that reovirus-induced mortality is reduced in mice inoculated at or after $8 \mathrm{~d}$ of age.

\section{Reovirus-Induced Disease Is Age-Dependent}

We monitored the weight gain of reovirus-inoculated mice as a quantitative surrogate measure of reovirus-induced disease. Mice were inoculated intracranially with $100 \mathrm{PFU} / \mathrm{g}$ T3D in PBS at 2, 4, 6, 8, 10, 15, or $20 \mathrm{~d}$ of age or PBS alone at $2 \mathrm{~d}$ of age and weighed daily for $20 \mathrm{~d}$. The mean weight gain of mice inoculated with reovirus at 2, 4, 6, and $8 \mathrm{~d}$ of age was significantly less than that of age-matched controls inoculated with PBS (Figure $\mathbf{1 b}-\mathbf{e}$ ). The mean weight gain of mice inoculated with reovirus at $10 \mathrm{~d}$ was significantly less than that of age-matched controls at early time points post inoculation (p.i.), but the differences were reduced at later time points (Figure 1f). Interestingly, the mean weights of mice inoculated with reovirus at 15 and $20 \mathrm{~d}$ of age were significantly greater than those of age-matched controls at later time points (Figure 1g,h). These data suggest that the capacity of reovirus to induce disease is diminished in mice inoculated at $10 \mathrm{~d}$ of age or older.

\section{Viral Loads in the Brain and Peripheral Organs Do Not Strictly Correlate with Disease Severity}

Disease severity is often proportional to the level of viral replication in vital organs. To determine whether the agedependent severity in reovirus disease is a function of viral load, we quantified viral titers in organs at 4,7 , and $10 \mathrm{~d}$ 

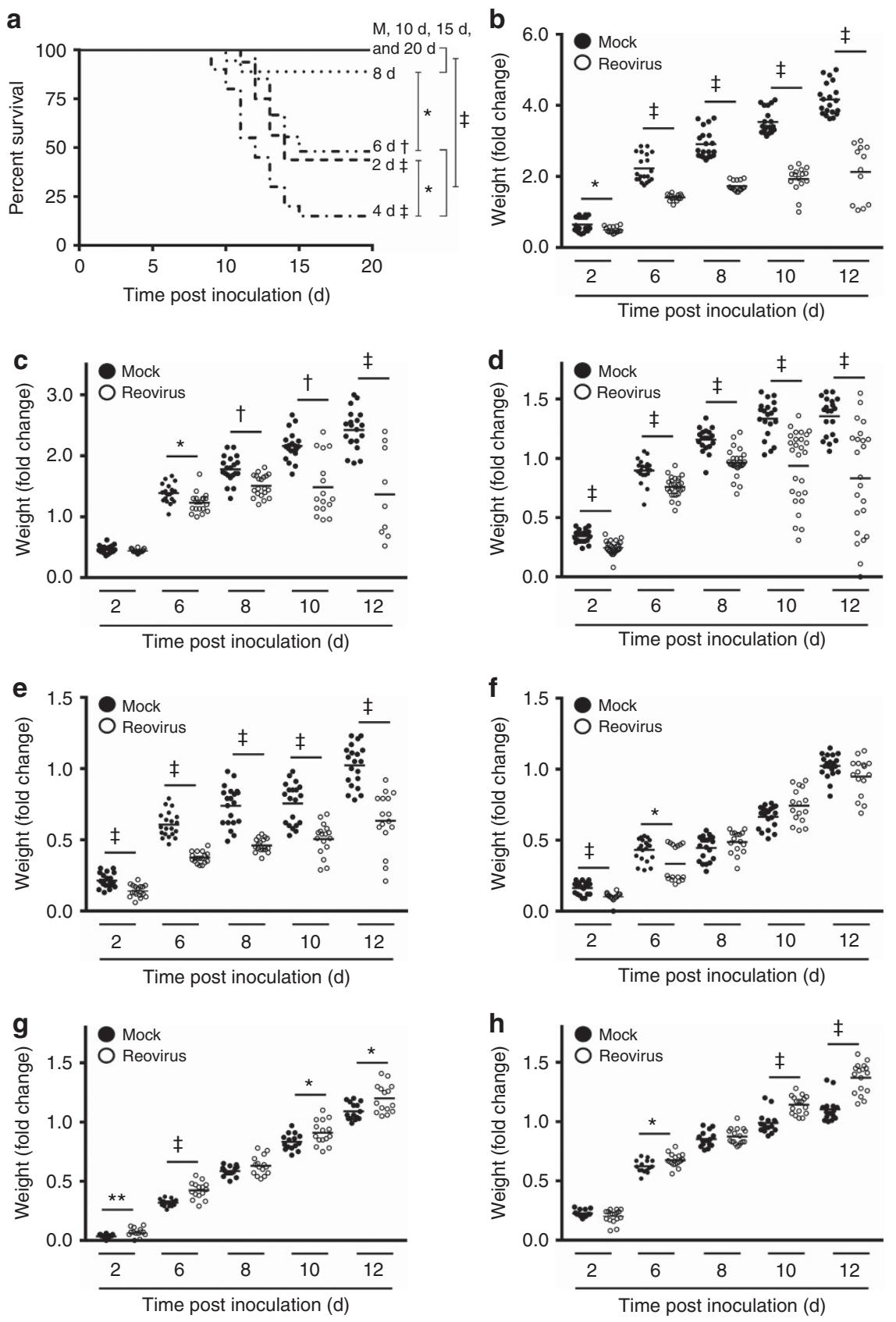

Figure 1. Survival and weight gain in mice following reovirus inoculation at various ages. WT mice were inoculated intracranially with reovirus T3D at $100 \mathrm{PFU} / \mathrm{g}$ at $2,4,6,8,10,15$, or $20 \mathrm{~d}$ of age or with PBS alone (mock; M) at $2 \mathrm{~d}$ of age. (a) Mice ( $n \geq 15$ per experimental group) were monitored for morbidity for $20 \mathrm{~d}$ and killed when moribund. Statistical analyses compared each curve to mock, to the next age of inoculation (e.g., 2 vs. $4 \mathrm{~d}$; 4 vs. 6 d, etc.), and between susceptible and nonsusceptible age groups (e.g., 2-6 vs. 8-20 d). ${ }^{*} P<0.05 ;{ }^{\dagger} p<0.005 ;{ }^{\ddagger} p<0.0001$ as determined by log-rank test. WT mice were inoculated intracranially with reovirus T3D at 100 PFU/g at (b) 2, (c) 4, (d) 6, (e) 8, (f) 10, (g) 15, or (h) 20 d of age and monitored for weight gain for $20 \mathrm{~d}$. Data are represented as fold change normalized to weight on the day of inoculation compared with agematched, mock controls at $2,6,8,10$, and $12 \mathrm{~d}$ p.i. ${ }^{*} P<0.05 ;{ }^{* *} P<0.01 ;{ }^{\dagger} P<0.005 ;{ }^{\ddagger} P<0.0001$ as determined by Mann-Whitney $U$-test. d p.i., day post inoculation; PBS, phosphate-buffered saline; PFU, plaque-forming unit; T3D, type 3 Dearing.

post-intracranial inoculation with T3D. The average peak viral loads in the brains of mice inoculated at 2 and $4 \mathrm{~d}$ of age were comparable (Figure 2). In contrast, the average peak viral load in the brains of mice inoculated at $6 \mathrm{~d}$ of age was lower compared with the average peak viral load in the brains of mice inoculated at a younger age. The average peak viral load in the brains of mice inoculated at 10 and $15 \mathrm{~d}$ of age was comparable to the average peak viral load in the brains of mice inoculated at $6 \mathrm{~d}$ of age. We calculated the area under the curve for each age of inoculation to test whether the cumulative viral loads in the brain for all three time points differed between mice inoculated at different ages. The areas 


\section{Articles | wu et al.}

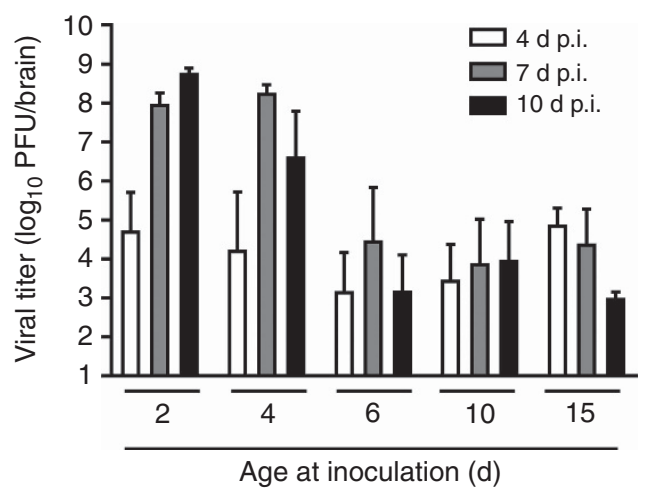

Figure 2. Viral loads in the brain following reovirus inoculation at various ages. WT mice were inoculated intracranially with reovirus T3D at $100 \mathrm{PFU} / \mathrm{g}$ at 2, 4, 6, 10, or $15 \mathrm{~d}$ of age. At 4, 7, and $10 \mathrm{~d}$ p.i., mice were killed, brains were resected, and viral loads were determined by plaque assay. $n=5-7$ mice per bar. $d$ p.i., day post inoculation; PFU, plaque-forming unit; T3D, type 3 Dearing.

Table 1. Viral dissemination to peripheral organs

\begin{tabular}{lccc}
\hline Age of inoculation $(d)$ & \multicolumn{3}{c}{ Time post inoculation $(d)$} \\
\hline 2 & 4 & 7 & 10 \\
4 & $3 / 6(50 \%)$ & $5 / 6(83 \%)$ & $6 / 6(100 \%)$ \\
6 & $3 / 6(50 \%)$ & $5 / 5(100 \%)$ & $5 / 6(83 \%)$ \\
10 & $1 / 5(20 \%)$ & $2 / 5(40 \%)$ & $0 / 7(0 \%)$ \\
15 & $3 / 6(50 \%)$ & $2 / 6(33 \%)$ & $0 / 6(0 \%)$ \\
\hline
\end{tabular}

WT mice were inoculated intracranially with reovirus T3D at $100 \mathrm{PFU} / \mathrm{g}$ at 2, 4, 6, 10 , or $15 \mathrm{~d}$ of age. Viral loads in the brain, heart, liver, spleen, and intestine were determined by plaque assay at 4, 7, and $10 \mathrm{~d}$ p.i. $n=5-7$ mice per condition. Numerals represent the number of mice with detectable titer in peripheral organs divided by the total number of mice assayed, and the resulting percentage of mice with detectable titer in peripheral organs in each group.

d, day; d p.i., day post inoculation; PFU, plaque-forming unit; T3D, type 3 Dearing; WT, wild type

under the curve of mice inoculated at $2 \mathrm{~d}$ of age $\left(5.5 \times 10^{14}\right)$ and $4 \mathrm{~d}$ of age $\left(5.0 \times 10^{13}\right)$ were markedly greater than the areas under the curve of mice inoculated at $6 \mathrm{~d}$ of age $\left(4.7 \times 10^{7}\right), 10 \mathrm{~d}$ of age $\left(4.2 \times 10^{7}\right)$, and $15 \mathrm{~d}$ of age $\left(2.2 \times 10^{8}\right)$. Although viral loads in the brains of mice inoculated at $6 \mathrm{~d}$ of age were comparable to those in mice inoculated at 10 or $15 \mathrm{~d}$ of age, mortality was significantly greater in mice inoculated at $6 \mathrm{~d}$ of age compared with mice inoculated at 10 or $15 \mathrm{~d}$ of age. These data suggest that viral loads in the brain do not strictly correlate with disease severity.

To determine whether the capacity to disseminate from the brain to other tissue sites in the host correlates with disease severity and, if so, whether this process is age-dependent, we quantified viral loads in the heart, spleen, liver, and intestine. We found that for each individual mouse, the virus was present either in all or none of the peripheral organs assayed. At $4 \mathrm{~d}$ p.i., approximately half of the mice inoculated at any age had detectable viral titers in peripheral organs with the exception of mice inoculated at $6 \mathrm{~d}$ of age, of which only one-fifth had detectable titers in peripheral organs (Table 1). At $7 \mathrm{~d}$ p.i., viral titers were detectable in peripheral organs of the majority of mice inoculated at 2 and $4 \mathrm{~d}$ of age compared with less than half the mice inoculated at 6,10 , or $15 \mathrm{~d}$ of age. At $10 \mathrm{~d}$ p.i., reovirus was detected in the majority of mice inoculated at 2 and $4 \mathrm{~d}$ of age, but no reovirus was detected in peripheral organs of mice inoculated at 6,10 , or $15 \mathrm{~d}$ of age. These data indicate that the capacity of reovirus to disseminate from the brain to peripheral organs is agedependent. However, neither viral load in the brain nor the presence of reovirus in peripheral organs alone explain the substantial disease severity observed in mice inoculated at $6 \mathrm{~d}$ of age.

\section{Reovirus Displays Enhanced Virulence in IFNAR ${ }^{-1-}$ but Not RAG2 $^{-1-}$ Mice}

To test the hypothesis that maturation of innate and adaptive immune responses contributes to age-dependent susceptibility to reovirus disease, we compared reovirus virulence following inoculation of WT, $\mathrm{IFNAR}^{-1-}$, and $\mathrm{RAG}^{-1-}$ mice at different ages. Mice deficient in expression of the IFN $\alpha / \beta$ receptor (IFNAR) lack an essential component of the antiviral innate immune response, whereas mice deficient in recombination-activating gene 2 (RAG2) lack functional B and $\mathrm{T}$ lymphocytes and are incapable of mounting adaptive immune responses. Mice were inoculated intracranially with T3D at 4 or $15 \mathrm{~d}$ of age and monitored for survival, weight gain, and clinical signs of neurologic diseases such as lethargy, seizures, ataxia, and paralysis (20) for $20 \mathrm{~d}$. The mean survival time for IFNAR ${ }^{-1-}$ mice inoculated with reovirus at $4 \mathrm{~d}$ of age was $8 \mathrm{~d}$, whereas the mean survival time for WT and RAG2 ${ }^{-1-}$ mice was 12 and $11 \mathrm{~d}$, respectively (Figure 3a). Only $48 \%$ of $\mathrm{IFNAR}^{-1-}$ mice inoculated with reovirus at $15 \mathrm{~d}$ of age survived compared with $92 \%$ of RAG2 ${ }^{-1-}$ mice and $100 \%$ of WT mice inoculated at $15 \mathrm{~d}$ of age (Figure $3 \mathrm{~b}$ ). All PBSinoculated mice survived. A majority of $\mathrm{IFNAR}^{-/-}$mice inoculated at either 4 or $15 \mathrm{~d}$ of age displayed reduced weight gain (Figure 3c,d) without neurological signs of illness. $\mathrm{RAG2}^{-1-}$ mice inoculated with reovirus displayed reduced weight gain when inoculated at $4 \mathrm{~d}$ of age but not when inoculated at $15 \mathrm{~d}$ of age, a trend similar to that of WT mice inoculated with reovirus at these ages (Figure 3e,f). These data suggest that the absence of a functional innate but not adaptive immune response prolongs the susceptibility of mice to reovirus-induced disease and raises the possibility that maturation of the IFN response contributes to age-dependent reovirus virulence.

\section{Viral Loads in the Brains of IFNAR ${ }^{-/-}$Mice Are Higher than Those In WT Mice When Inoculated at an Older Age}

On the basis of the increased mortality in IFNAR ${ }^{-1-}$ mice at older ages of inoculation, we hypothesized that reovirus produces higher titers in IFNAR ${ }^{-/-}$mice when inoculated at ages at which WT mice are no longer susceptible to reovirus disease. To test this hypothesis, WT, IFNAR ${ }^{-1-}$, and RAG2 $2^{-1-}$ mice were inoculated intracranially with $\mathrm{T} 3 \mathrm{D}$ at the susceptible 

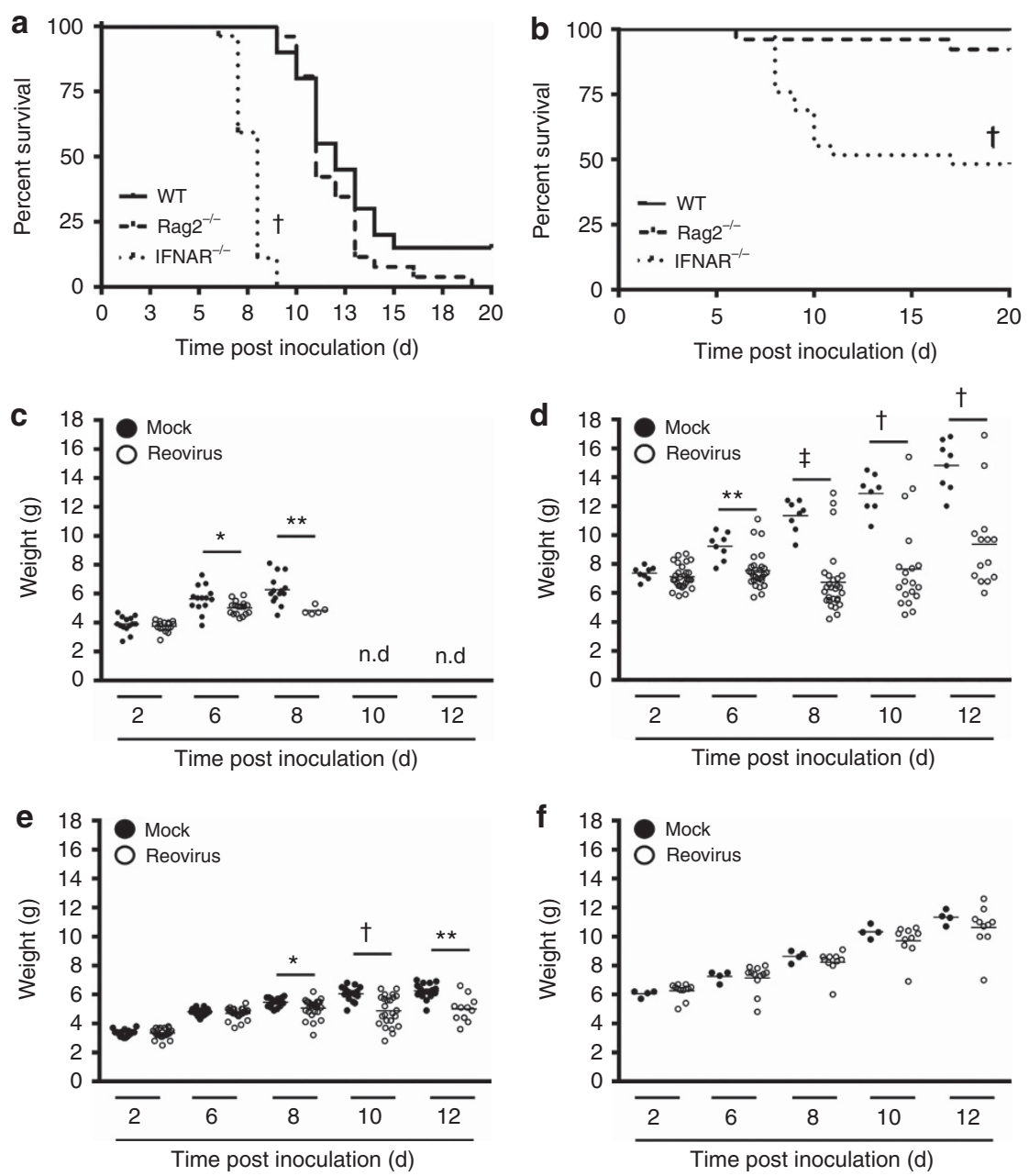

Figure 3. Survival and weight gain of reovirus-infected WT, IFNAR ${ }^{-/-}$, and $\mathrm{RAG}^{-/-}$mice. WT, IFNAR ${ }^{-/-}$, and $\mathrm{RAG}^{-/-}$mice were inoculated intracranially with reovirus T3D at $100 \mathrm{PFU} / \mathrm{g}$ at either 4 or $15 \mathrm{~d}$ of age and monitored for morbidity for $20 \mathrm{~d}$. Mice were killed when moribund. Survival of mice inoculated at (a) 4 and (b) 15 d of age. $N \geq 23$ per experimental group. ${ }^{\dagger} P<0.001$ as determined by log-rank test compared with WT mice. Weights of IFNAR ${ }^{-1-}$ mice inoculated at (c) 4 or (d) $15 \mathrm{~d}$ of age. Weights of RAG2 ${ }^{-/-}$mice inoculated at (e) 4 or (f) $15 \mathrm{~d}$ of age. $N \geq 15$ per experimental group. ${ }^{*} P<0.05 ;{ }^{* *} P<0.01 ;{ }^{\dagger} P<0.005$; and ${ }^{\ddagger} P<0.0001$ as determined by Mann-Whitney $U$-test. IFNAR, interferon a/ $\beta$ receptor; RAG, recombination-activating gene; $\mathrm{T} 3 \mathrm{D}$, type 3 Dearing.

age of $4 \mathrm{~d}$ and the nonsusceptible age of $15 \mathrm{~d}$, and viral loads in organs were determined at 4,7 , and $10 \mathrm{~d}$ p.i. Viral loads in the brains of WT, IFNAR ${ }^{-/-}$, and RAG2 ${ }^{-/-}$mice inoculated at $4 \mathrm{~d}$ of age did not differ significantly on 4 and $7 \mathrm{~d}$ p.i (Figure 4a). However, viral loads in the brains of $\mathrm{RAG2}^{-1-}$ mice were significantly higher than those in the brains of WT mice at $10 \mathrm{~d}$ p.i. We were unable to perform statistics comparing viral loads in the brains of IFNAR ${ }^{-1-}$ mice at $10 \mathrm{~d}$ p.i. because of the lack of replicates. However, the viral load in the brain of the single surviving mouse approximated the average load of $\mathrm{RAG}^{-1-}$ mice inoculated at that age. At all time points tested, viral loads in the brains of IFNAR ${ }^{-1-}$ mice were significantly higher than those in the brains of WT mice inoculated at $15 \mathrm{~d}$ of age (Figure 4b). No significant differences in viral load were detected between the brains of WT and $\mathrm{RAG}^{-/-}$mice inoculated at $15 \mathrm{~d}$. These data suggest that adaptive immune responses function in the clearance of reovirus from the brains of younger mice, whereas IFN-mediated innate immune responses control viral replication in the brains of older mice. The lack of overwhelmingly significant immune-related differences in viral loads in the brains of mice inoculated at 4 $\mathrm{d}$ of age suggests that disease severity and mortality at this age of inoculation are not directly related to the modulation of viral replication by immune responses in the brain.

\section{IFN Controls Viral Dissemination to Peripheral Organs in Both Younger and Older Mice}

To determine the function of innate and adaptive immune responses in controlling viral dissemination from the site of inoculation to sites of secondary replication, viral loads were quantified in organs of WT, IFNAR ${ }^{-1-}$, and $\mathrm{RAG}^{-1-}$ mice inoculated at 4 and $15 \mathrm{~d}$ of age (Figure $4 \mathrm{c}$-j). Contrary to WT mice (Table 1), reovirus was detected in all peripheral organs in both strains of immune-deficient mice 

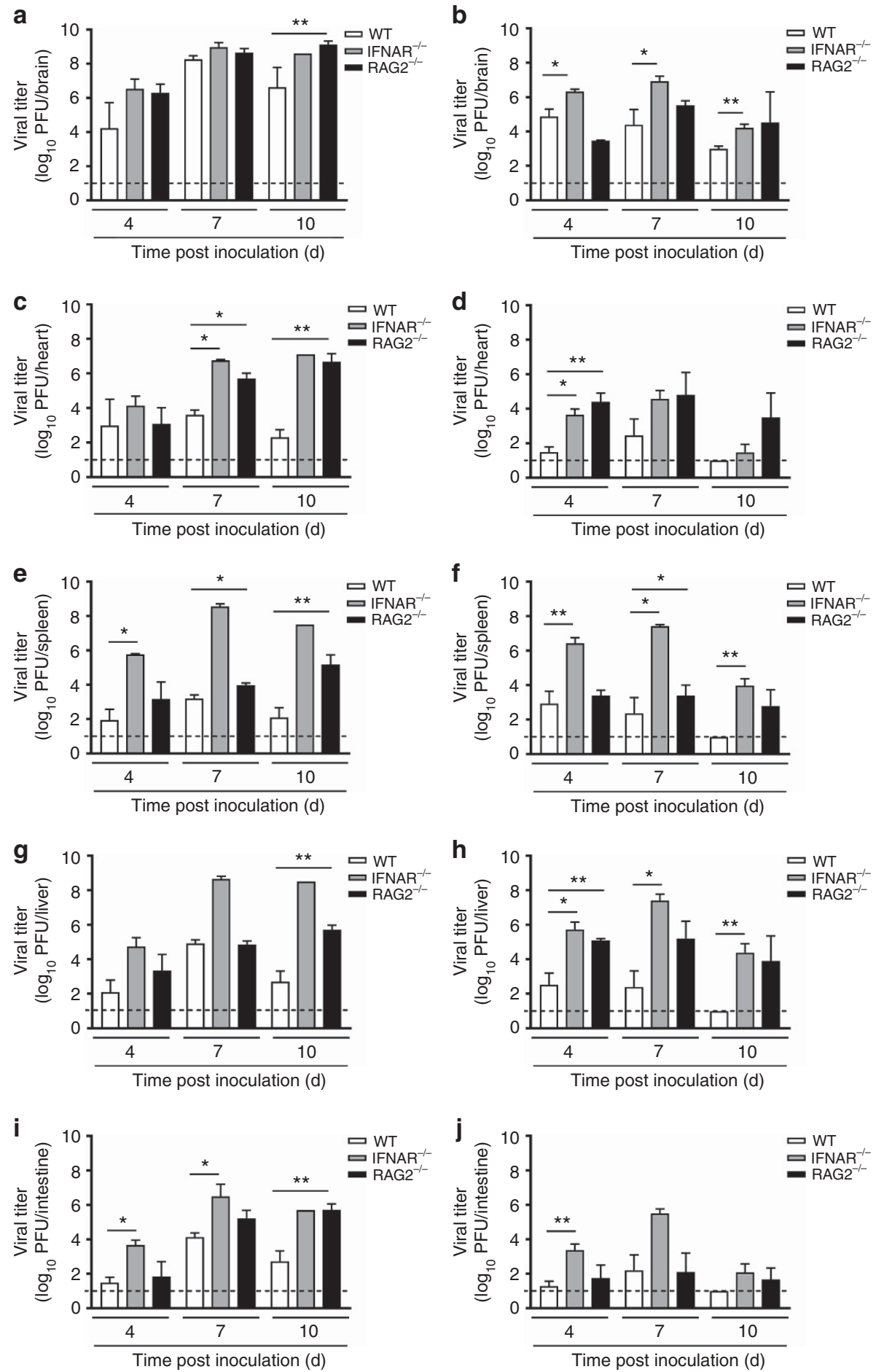

Figure 4. Viral loads in reovirus-infected WT, IFNAR ${ }^{-/-}$, and $\mathrm{RAG}^{-/-}$mice. WT, IFNAR ${ }^{-/-}$, and $\mathrm{RAG}^{-/-}$mice were inoculated intracranially with reovirus T3D at $100 \mathrm{PFU} / \mathrm{g}$ at either 4 or $15 \mathrm{~d}$ of age. At 4, 7, and $10 \mathrm{~d}$ p.i., mice were killed, organs were resected, and viral loads were determined. Viral loads in the brains of mice inoculated at (a) 4 or (b) $15 \mathrm{~d}$ of age, in the hearts of mice inoculated at (c) 4 or (d) $15 \mathrm{~d}$ of age, in the spleens of mice inoculated at (e) 4 or (f) $15 \mathrm{~d}$ of age, in livers of mice inoculated at (g) 4 or (h) $15 \mathrm{~d}$ of age, and in the intestines of mice inoculated at (i) 4 or (j) $15 \mathrm{~d}$ of age. $N=2-10$ except IFNAR ${ }^{-1-}$ mice $10 \mathrm{~d}$ p.i., for which $n=1$ due to the low survival rate at that time point. Dotted lines represent the limit of detection. ${ }^{*} P<0.05 ;{ }^{* *} P<0.01$ as determined by Mann-Whitney $U$-test. $d$ p.i., day post inoculation; IFNAR, interferon $a / \beta$ receptor; PFU, plaque-forming unit; RAG, recombination-activating gene; T3D, type 3 Dearing.

at all time points tested (data not shown). Overall, viral loads in peripheral organs of IFNAR $^{-1-}$ mice were increased compared with those in WT mice (Figure $4 \mathbf{c}-\mathbf{j}$ ). Viral loads in the heart and spleen of RAG2 ${ }^{-/-}$mice inoculated at $4 \mathrm{~d}$ of age were also increased compared with those in WT mice at $7 \mathrm{~d}$ p.i. and in all organs at $10 \mathrm{~d}$ p.i. $\mathrm{RAG}^{-1-}$ mice 


\section{Mechanisms of reovirus age restriction Articles}

inoculated at $15 \mathrm{~d}$ of age displayed modestly increased viral loads in the heart and liver but not in the spleen and intestine at $4 \mathrm{~d}$ p.i., and the differences became smaller at later times (Figure $4 \mathbf{4 d}, \mathbf{f}, \mathbf{h}, \mathbf{j})$. Peak viral loads in all organs of IFNAR ${ }^{-1-}$ mice inoculated at $4 \mathrm{~d}$ of age coincide with the sharp reduction in survival of IFNAR ${ }^{-1-}$ mice (Figure 3a), whereas differences in weight gain and survival of WT and RAG2 $2^{-1-}$ mice inoculated at $4 \mathrm{~d}$ of age appear at later times post infection when viral loads in both the brain and peripheral organs of RAG2 ${ }^{-/-}$mice are significantly greater than those in WT mice (Figure 4a,c,e,g,i). Interestingly, viral loads in IFNAR $^{-1-}$ mice inoculated at $4 \mathrm{~d}$ of age increased throughout infection until death, whereas viral loads in mice inoculated at $15 \mathrm{~d}$ of age peaked at $7 \mathrm{~d}$ p.i., followed by a decline at $10 \mathrm{~d}$ p.i., consistent with the enhanced survival of $\mathrm{IFNAR}^{-/-}$ mice inoculated at this age. Together, these results suggest that IFN has an important role in controlling systemic dissemination and replication at secondary sites in mice of both susceptible and nonsusceptible ages at all time points assessed. Adaptive responses function later during infection of mice inoculated at a susceptible age and likely contribute to viral clearance.

\section{Reovirus Tropism Is Unaltered in the Brains of Immune- Deficient Mice}

To determine whether differences in brain pathology link mortality to altered immune responses, the right hemispheres of brains that matched as closely as possible for viral load were processed for histology. Consecutive sections were stained with hematoxylin and eosin, with polyclonal reovirus antiserum to detect viral antigen, or with a monoclonal antibody specific for the cleaved (activated) form of caspase-3 to detect cells undergoing apoptosis-the primary mechanism of neuronal cell death following reovirus infection (19). Reovirus antigen was detected in the hippocampus, thalamus, and cortex (Figure 5a) as well as the cerebellum and hindbrain (Figure 5b) of brains resected at $7 \mathrm{~d}$ p.i. from mice of all three strains inoculated with reovirus at $4 \mathrm{~d}$ of age. Although the overall staining intensity varied, no qualitative differences were found in viral tropism. Staining for the activated form of caspase-3 was modest in all sections analyzed and localized with reovirus staining, consistent with the pattern of reovirus-induced tissue injury $(8,19,21)$. Histological analysis of brains resected from mice inoculated with reovirus at $15 \mathrm{~d}$ of age at $7 \mathrm{~d}$ p.i. showed substantially reduced levels of reovirus antigen-positive cells (data not shown). Staining was restricted to small areas within the thalamus and surrounding the lateral ventricles. These areas of the brain also displayed low levels of activated caspase-3 staining (data not shown).

Apoptosis Is Unaltered in the Brains of Immune-Deficient Mice To determine whether quantitative age- and immunedependent differences exist in reovirus-induced apoptosis, protein lysates from the brains of three individual mice of each strain resected $7 \mathrm{~d}$ p.i. were resolved by SDS-PAGE and

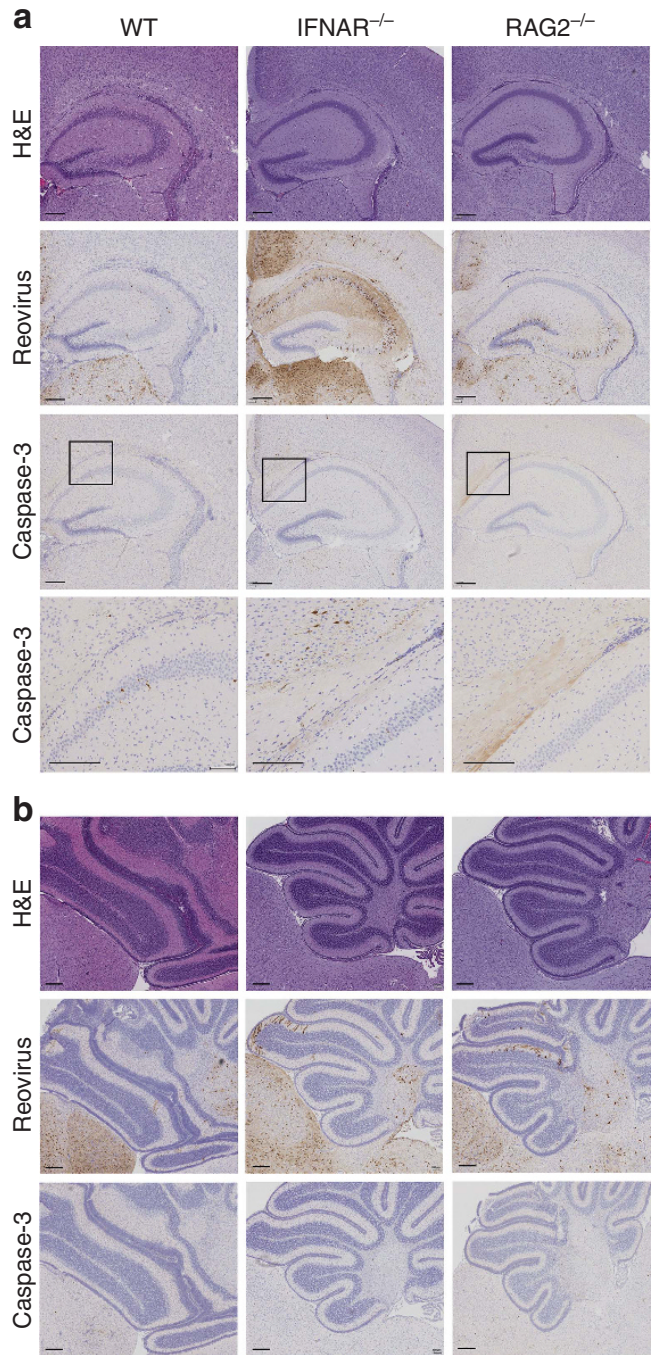

Figure 5. Reovirus tropism in the brains of WT, IFNAR ${ }^{-/-}$, and RAG2 $2^{-1-}$ mice. WT, IFNAR ${ }^{-1-}$, and RAG2 ${ }^{-/-}$mice were inoculated intracranially with reovirus T3D at $100 \mathrm{PFU} / \mathrm{g}$ at $4 \mathrm{~d}$ of age. At $7 \mathrm{~d}$ p.i., mice were killed, brains were removed, the left hemispheres were homogenized for the determination of viral titer, and the right hemispheres were processed for immunohistochemistry. Consecutive coronal sections of the brain were stained with $\mathrm{H} \& \mathrm{E}$, reovirus antiserum, or an anticleaved caspase- 3 antibody. Representative sections of the brains are shown. (a) Hippocampal region stained with $\mathrm{H} \& \mathrm{E}$ or polyclonal reovirus antiserum and a higher-magnification image of the boxed inset stained for cleaved caspase-3 (scale bars, $200 \mu \mathrm{m}$ ). (b) Cerebellum and the hindbrain. Sections shown are from a WT, IFNAR ${ }^{-/-}$, and a RAG2 $2^{-1-}$ mouse with a viral load of $3.9 \times 10^{8} \mathrm{PFU} /$ brain, $3.8 \times 10^{9} \mathrm{PFU} /$ brain, and $1.2 \times 10^{9} \mathrm{PFU} /$ brain, respectively (scale bars, $200 \mu \mathrm{m}$ ). $\mathrm{d}$ p.i., day post inoculation; H\&E, hematoxylin and eosin; IFNAR, interferon $\alpha / \beta$ receptor; PFU, plaque-forming unit; RAG, recombination-activating gene; T3D, type 3 Dearing.

immunoblotted using an antibody specific for cleaved caspase- 3 and an antiserum specific for actin as a loading control. The intensity of the cleaved caspase- 3 signal was normalized to the intensity of the actin signal. The overall magnitude of apoptosis in the brains of $\mathrm{IFNAR}^{-1-}$ and $\mathrm{RAG}^{-1-}$ mice inoculated at $4 \mathrm{~d}$ of age (Figure $6 \mathrm{a}, \mathrm{c}$ ) and at $15 \mathrm{~d}$ of age (Figure $\mathbf{6 b}, \mathbf{d}$ ) did not differ statistically. 


\section{Articles | wu et al.}

a
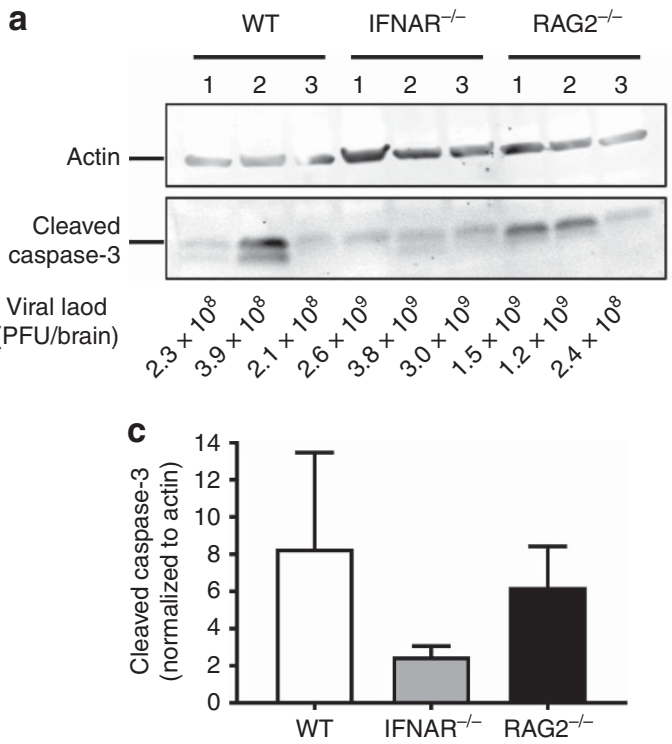

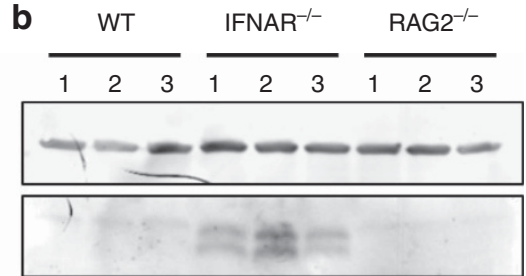

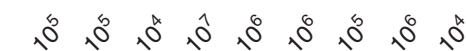

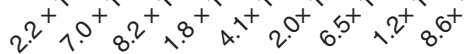

d

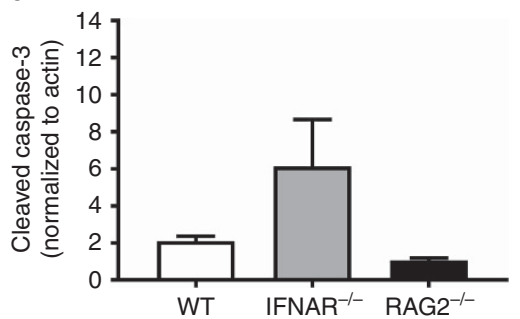

Figure 6. Quantification of apoptosis in the brains of WT, IFNAR ${ }^{-1-}$, and RAG2 ${ }^{-1-}$ mice. Mice were inoculated intracranially with reovirus T3D at $100 \mathrm{PFU} / \mathrm{g}$ at either $(\mathbf{a}, \mathbf{c}) 4$ or $(\mathbf{b}, \mathbf{d}) 15 \mathrm{~d}$ of age. Protein lysates from the brains of three T3D-infected WT, IFNAR ${ }^{-/-}$, or RAG2 $2^{-/-}$mice resected at $7 \mathrm{~d}$ p.i. were resolved by SDS-PAGE. (a,b) Lysates were immunoblotted using antisera specific for actin (top) and cleaved caspase-3 (bottom). Viral loads are listed for each sample. (c,d) Quantification of the band intensity of cleaved caspase-3 normalized to actin. $d$ p.i., day post inoculation; IFNAR, interferon $\alpha / \beta$ receptor; PFU, plaque-forming unit; RAG, recombination-activating gene; T3D, type 3 Dearing.

\section{DISCUSSION}

In this study, we determined the precise timing of the age restriction to reovirus virulence in $\mathrm{C} 57 \mathrm{BL} / 6 \mathrm{~J}$ mice based on mortality and determination of weight gain as a surrogate marker for reovirus-induced disease. We found that the age restriction for reovirus mortality and disease lies between 6 and $10 \mathrm{~d}$ of age. We were surprised to find that a lower proportion of mice inoculated at $4 \mathrm{~d}$ of age survived compared with mice inoculated at $2 \mathrm{~d}$ of age. This finding might be attributed to experimental variability caused by a suboptimal nurturing by the dam. Mice inoculated intracranially with serotype 3 reovirus strains likely succumb to encephalitis rather than to disease at other sites of infection, as a reovirus mutant incapable of disseminating systemically is as virulent as WT virus following intracranial inoculation (22). Interestingly, there was a significant increase in weight gain following inoculation with reovirus at 15 and $20 \mathrm{~d}$ of age relative to PBS-inoculated controls. It is possible that the virus-mediated increase in weight gain occurs as a consequence of virus-induced damage to the ventromedial hypothalamus, which is associated with increased appetite (23-25). Histological examination of brain tissue from mice infected at additional ages will provide more information about the age-dependent differences in reovirus neural tropism and pathology.

We thought it possible that age-related disease severity might track with virus titers in target tissues. Our results indicate that viral replication occurred even in mice that did not display overt neurological signs of infection. Brains of mice inoculated at $6 \mathrm{~d}$ of age harbored viral loads that were similar to those in the brains of mice inoculated at nonsusceptible ages; yet, survival rates and weight gain were comparable to mice inoculated at susceptible ages. Reovirus disseminated systemically in mice inoculated at susceptible ages, whereas systemic dissemination was limited in mice inoculated at nonsusceptible ages. Thus, we conclude that viral titers in the brain do not strictly correlate with susceptibility to reovirus-induced disease.

We used immune-deficient mice to investigate whether maturation of innate or adaptive immune responses contributes to the age restriction of reovirus disease. Our results indicate that IFN functions in controlling viral replication in mice of both susceptible and nonsusceptible ages, whereas adaptive immune responses are particularly important in controlling replication at later times post infection in mice of susceptible age. The increased susceptibility of older IFNAR ${ }^{-/-}$ mice to reovirus infection suggests that age-dependent maturation of IFN responses contributes to the age-related virulence of reovirus. Some $\mathrm{IFNAR}^{-/-}$mice had signs of encephalitis, but others died quickly after the onset of illness. A previous study describes intestinal perforation, bacterial sepsis, and acute hepatitis as causes of death in $\mathrm{IFNAR}^{-1-}$ mice inoculated with reovirus at $2 \mathrm{~d}$ of age (26).

Besides contributing to age-dependent susceptibility to reovirus infection, the type-I IFN response functions in the age-dependent susceptibility to infection with herpes simplex virus (HSV-1). Lower basal levels of IFNAR are expressed in the choroid plexus of uninfected newborn mice compared with adults. Concordantly, the adult choroid plexus is less susceptible to infection with HSV-1 relative to the 
newborn brain. Similar to our findings with reovirus, HSV-1 susceptibility was restored in the brains of adult $\mathrm{IFNAR}^{-1-}$ mice (27).

Cells of the innate immune system may also contribute directly to the age-dependent susceptibility to reovirus central nervous system disease. Microglia, the resident macrophage cells in the brain, modulate the immune response to brain infections by secreting inflammatory cytokines such as interleukin (IL)-1 $\alpha$, IL-6, and tumor necrosis factor- $\alpha$ (28). Microglia are virtually absent from the mouse hippocampus at birth, but the numbers of these cells increase markedly between 5 and $10 \mathrm{~d}$ of age and peak at $15 \mathrm{~d}$ of age (29). Microglial activation and proinflammatory cytokine production in the brain decrease with age under normal conditions and in response to stimulation with lipopolysaccharide $(30,31)$, suggesting that the inflammatory response to viral infections of the brain is muted later in life. Consistent with this idea, the production of the proinflammatory cytokine IL- $1 \alpha$ is increased in the brains of reovirus-infected newborn mice compared with adults and coincides with nervous tissue injury that precedes encephalitis (32). Our finding that the absence of RAG2 expression does not affect the timing of the age restriction to reovirus disease is consistent with the kinetics of adaptive immune effector maturation, which is initiated after the first month of life (33) and, thus, outside the interval during which reovirus susceptibility diminishes.

The results from this study define an age at which mice become refractory to reovirus disease and provide evidence that maturation of innate immune responses contributes to the mechanism of age restriction. These findings suggest that innate immune maturity influences diverse types of neural insults.

\section{ACKNOWLEDGMENTS}

We thank members of the Dermody lab for helpful discussions and Laurie Silva and Gwen Taylor for a critical review of the manuscript, the Vanderbilt Division of Animal Care for animal husbandry, and Kelli Boyd and colleagues from the Vanderbilt Translational Pathology Shared Resource for assistance.

\section{STATEMENT OF FINANCIAL SUPPORT}

This work was supported by Public Health Service awards T32 HD060554 (A.G.W.), F31 DK108562 (J.J.B.), and R01 Al38296 (T.S.D.). Additional support was provided by the Elizabeth B. Lamb Center for Pediatric Research.

Disclosure: The authors declare no competing financial interests.

\section{REFERENCES}

1. Griffin DE. Emergence and re-emergence of viral diseases of the central nervous system. Prog Neurobiol 2010;91:95-101.

2. Griffin DE. Viral encephalomyelitis. PLoS Pathog 2011;7:e1002004.

3. Tyler KL, McPhee DA, Fields BN. Distinct pathways of viral spread in the host determined by reovirus S1 gene segment. Science 1986;233: $770-4$.

4. Morrison LA, Sidman RL, Fields BN. Direct spread of reovirus from the intestinal lumen to the central nervous system through vagal autonomic nerve fibers. Proc Natl Acad Sci USA 1991;88:3852-6.

5. Antar AAR, Konopka JL, Campbell JA, et al. Junctional adhesion molecule-A is required for hematogenous dissemination of reovirus. Cell Host Microbe 2009;5:59-71.
6. Pruijssers AJ, Dermody TS Reovirus. In: Reiss CS, ed. Neurotropic Viral Infections. 2nd edn, New York, NY: Springer Nature; 2016:337-360.

7. Weiner HL, Powers ML, Fields BN. Absolute linkage of virulence and central nervous system cell tropism of reoviruses to viral hemagglutinin. J Infect Dis 1980;141:609-16.

8. Danthi P, Coffey CM, Parker JS, Abel TW, Dermody TS. Independent regulation of reovirus membrane penetration and apoptosis by the mul phi domain. PLoS Pathog 2008;4:e1000248.

9. Danthi P, Pruijssers AJ, Berger AK, Holm GH, Zinkel SS, Dermody TS. Bid regulates the pathogenesis of neurotropic reovirus. PLoS Pathog 2010;6:e1000980.

10. Margolis G, Kilham L, Gonatos N. Reovirus type III encephalitis: observations of virus-cell interactions in neural tissues. I. Light microscopy studies. Lab Invest 1971;24:91-109.

11. Raine CS, Fields BN. Ultrastructural features of reovirus type 3 encephalitis. J Neuropathol Exp Neurol 1973;32:19-33.

12. Tardieu M, Powers ML, Weiner HL. Age dependent susceptibility to Reovirus type 3 encephalitis: role of viral and host factors. Ann Neurol 1983;13:602-7.

13. Ivashkiv LB, Donlin LT. Regulation of type I interferon responses. Nat Rev Immunol 2014;14:36-49.

14. Goody RJ, Beckham JD, Rubtsova K, Tyler KL. JAK-STAT signaling pathways are activated in the brain following reovirus infection. J Neurovirol 2007;13:373-83.

15. Kobayashi T, Antar AA, Boehme KW, et al. A plasmid-based reverse genetics system for animal double-stranded RNA viruses. Cell Host Microbe 2007;1:147-57.

16. Furlong DB, Nibert ML, Fields BN. Sigma 1 protein of mammalian reoviruses extends from the surfaces of viral particles. J Virol 1988;62:246-56.

17. Virgin HW, Dermody TS, Tyler KT Cellular and humoral immunity to reovirus infection. In: Tyler KT, Oldstone MBA, eds. Curr Top Microbiol Immunol. Berlin: Springer; 1998:147-61.

18. Tyler KL, Bronson RT, Byers KB, Fields B. Molecular basis of viral neurotropism: experimental reovirus infection. Neurology 1985;35:88-92.

19. Pruijssers AJ, Hengel H, Abel TW, Dermody TS. Apoptosis induction influences reovirus replication and virulence in newborn mice. J Virol 2013;87:12980-9.

20. Tyler KL Pathogenesis of reovirus infections of the central nervous system. In: Tyler KL, Oldstone MA, eds. Reoviruses II Cytopathogenicity and Pathogenesis. Berlin, Heidelberg: Springer; 1998:93-124.

21. Richardson-Burns SM, Tyler KL. Regional differences in viral growth and central nervous system injury correlate with apoptosis. J Virol 2004;78: 5466-75.

22. Boehme KW, Guglielmi KM, Dermody TS. Reovirus nonstructural protein sigmals is required for establishment of viremia and systemic dissemination. Proc Natl Acad Sci USA 2009;106:19986-91.

23. Reyes-Vazquez C, Prieto-Gomez B, Dafny N. Interferon modulates central nervous system function. Brain Res 2012;1442:76-89.

24. Dafny N, Gillman MA, Lichtigfeld FJ. Cholecystokinin: induced suppression of feeding in fed, fasting and hypothalamic island rats. Brain Res Bull 1988;21:225-31.

25. King BM. The rise, fall, and resurrection of the ventromedial hypothalamus in the regulation of feeding behavior and body weight. Physiol Behav 2006;87:221-44.

26. Dionne KR, Galvin JM, Schittone SA, Clarke P, Tyler KL. Type I interferon signaling limits reoviral tropism within the brain and prevents lethal systemic infection. J Neurovirol 2011;17:314-26.

27. Wilcox DR, Folmsbee SS, Muller WJ, Longnecker R. The type I interferon response determines differences in choroid plexus susceptibility between newborns and adults in herpes simplex virus encephalitis. mBio 2016; 7 : e00437-16.

28. Rock RB, Gekker G, Hu S, et al. Role of microglia in central nervous system infections. Clin Microbiol Rev 2004;17:942-64.

29. Kim I, Mlsna LM, Yoon S, et al. A postnatal peak in microglial development in the mouse hippocampus is correlated with heightened sensitivity to seizure triggers. Brain Behav 2015;5:e00403. 


\section{Articles | wu et al.}

30. Ferrazzano P, Chanana V, Uluc K, et al. Age-dependent microglial activation in immature brains after hypoxia- ischemia. CNS Neurol Disord Drug Targets 2013;12:338-49.

31. Inamizu T, Chang MP, Makinodan T. Influence of age on the production and regulation of interleukin-1 in mice. Immunology 1985;55:447-55.
32. Derrien M, Fields BN. Reovirus type 3 clone 9 increases interleukin-1 level in the brain of neonatal, but not adult, mice. Virology 1999;257: $35-44$.

33. Landreth KS. Critical windows in development of the rodent immune system. Hum Exp Toxicol 2002;21:493-8. 